\title{
Characterization and oil recovery enhancement by a polymeric nanogel combined with surfactant for sandstone reservoirs
}

\author{
Mustafa Almahfood ${ }^{1,2} \cdot$ Baojun Bai $^{1}$
}

Received: 19 April 2020 / Accepted: 28 September 2020 / Published online: 12 November 2020

(c) The Author(s) 2020

\begin{abstract}
The characterization and enhanced oil recovery mechanisms of a nanosized polymeric cross-linked gel are presented herein. A negatively charged nanogel was synthesized using a typical free radical suspension polymerization process by employing 2-acrylamido 2-methyl propane sulfonic acid monomer. The synthesized nanogel showed a narrow size distribution with one peak pointing to a predominant homogeneous droplet size. The charged nanogels were also able to adsorb at the oil-water interfaces to reduce interfacial tension and stabilize oil-in-water emulsions, which ultimately improved the recovered oil from hydrocarbon reservoirs. In addition, a fixed concentration of negatively charged surfactant (sodium dodecyl sulfate or SDS) was combined with different concentrations of the nanogel. The effect of the nanogels combined with surfactant on sandstone core plugs was examined by running a series of core flooding experiments using multiple flow patterns. The results show that combining nanogel and SDS was able to reduce the interfacial tension to a value of $6 \mathrm{Nm} / \mathrm{m}$. The core flooding experiments suggest the ability of the nanogel, both alone and combined with SDS, to improve the oil recovery by a factor of $15 \%$ after initial seawater flooding.
\end{abstract}

Keywords Nanogel $\cdot$ Polymeric nanogel $\cdot$ Enhanced oil recovery

$\begin{array}{ll}\text { Abbreviations } \\ \text { CMC } & \text { Critical micelle concentration } \\ \text { DSW } & \text { Diluted seawater } \\ \text { IFT } & \text { interfacial tension }(\mathrm{mN} / \mathrm{m}) \\ \text { OOIC } & \text { Original oil in core } \\ \text { NG } & \text { Nanogel } \\ \text { ppm } & \text { Parts per million } \\ \text { PV } & \text { Pore volume } \\ \text { SDS } & \text { Sodium dodecyl sulfate } \\ \text { SW } & \text { Seawater } \\ F_{\mathrm{r}} & \text { Resistance factor } \\ F_{\mathrm{rrw}} & \text { Residual resistance factor of water } \\ S_{\mathrm{oi}} & \text { Initial oil saturation } \\ S_{\mathrm{wi}} & \text { Initial water saturation }\end{array}$

Edited by Yan-Hua Sun

Baojun Bai

baib@mst.edu

1 Missouri University of Science and Technology, Rolla, MO 65401, USA

2 EXPEC Advanced Research Center, Saudi Aramco, Dhahran, Saudi Arabia

\section{Introduction}

Most oilfields around the world have already reached or will soon reach the phase where the oil production rate is approaching the decline period (Hendraningrat et al. 2013). Thus, one of the major challenges that face the oil industry today is how to delay the abandonment of current fields by reducing excess water production and extracting more oil economically. Enhanced oil recovery (EOR) applications are generally implemented in oilfields to enhance oil recovery and reduce water production. Chemical-based EOR methods (thermal, gas, and alkine/surfacant/polymer (ASP) methods) can improve oil recovery through five major mechanisms: (1) interfacial tension reduction, (2) wettability modification toward a water-wet state, (3) conformance control improvement for better sweep efficiency, (4) emulsifying crude oil, and (5) foam generation (Binks et al. 2007; Thomas 2008; Pal et al. 2018). Gel treatments have been proved to be a cost-effective method for conformance control improvements (Bai et al. 2013). Different particle gels have been proposed to enhance oil recovery and control excess water production such as preformed particle gels (PPG) (Bai et al. 2007), micro-gels (Rousseau et al. 2005), temperaturesensitive gels which are commonly known as bright water 
(Frampton et al. 2004), and pH-sensitive gels (Al-Anazi et al. 2002). Nanosized cross-linked polymeric particles known as nanogels (NGs) are newly developed particles in EOR applications. They are defined as base fluids with nanosized particles that have an average particle size of less than $100 \mathrm{~nm}$ (Sun et al. 2017). They are, also, known for their easy injection process due to their small size, which is much smaller than the diameter of the pore throats in oil reservoirs (Qiu 2010). They are able to mobilize residual oil, which enhances oil recovery by mainly reducing the interfacial tension (Lenchenkov et al. 2016).

In contrast to conventional in-depth plugging agents such as preformed particle gels (PPG) and in situ gels, nanogels are characterized by low viscosity (Moraes et al. 2011; Bai et al. 2013). Also, nanogels can reduce the interfacial tension by adsorbing at the oil-water interface, which stabilizes oil-in-water emulsions, leading to improvement of the recovered oil from reservoirs (Geng et al. 2018a; Almahfood et al. 2020). Suleimanov and Veliyev (2017) showed that the oil recovery from a sandstone reservoir has increased by $6 \%$ after nanogel flooding, compared to gel treatment without nanosized particles.

Multiple experimental studies have concluded that nanogels can adsorb and form a blockage in porous media, which reduces the relative permeability of water (Almohsin et al. 2014). However, the surface charge of nanogels can eliminate to some degree aggregation of dispersed nanogels in water, which enhances their stability during transport in porous media. Surface charges also affect the arrangement and adsorption of nanogels at rock surfaces (Johnson and Lenhoff 1996). In general, nanogels are attracted to rock surfaces with opposite charges. It is extremely crucial to understand the interactions between nanogels and rock surfaces to better explain the permeability reduction mechanisms and nanogel transportation in porous media. Since sandstone reservoirs are characterized by negatively charged surfaces (Nasralla et al. 2013), transportation of charged nanogels in porous media and adsorption at the rock-fluid interface are greatly impacted.

Additionally, the employment of conventional chemical processes such as surfactant and polymer flooding was widely discussed in the literature. Surfactant flooding has played an essential role in enhanced oil recovery processes over the years due to its effectiveness in reducing oil-water interfacial tension, modifying the wettability of the oil phase toward a water-wet state and emulsifying crude oil (Green et al. 1998; Johannessen and Spildo 2013; Xu et al. 2020). Due to the above-mentioned features of both nanogels and surfactants, combining nanogels and surfactants together in oil fields is predicted to enhance their potential by greatly reducing the interfacial tension that leads to recovery of higher amounts of oil.
The interactions between surfactants and nanosized particles can lead to a considerable change in the surface activity of surfactants. Multiple layers of surfactant and nanoparticle can be formed by the strong attraction between surfactant and nanoparticle molecules. Thus, nanogels are added to surfactants to combine all recovery mechanisms and eliminate the drawbacks associated with each method when used individually. To the best of our knowledge after a comprehensive literature review about the combination of nanosized particles and surfactants in our previous work (Almahfood and Bai 2018), no studies have been reported on the effect of polymeric cross-linked nanogels combined with surfactants in EOR applications.

In this work, a negatively charged nanogel was synthesized using a free radical suspension polymerization process. The effect of adding a negatively charged surfactant (SDS) to the synthesized nanogel was demonstrated by measuring the interfacial tension between the combined dispersion and light mineral oil. Additionally, the combined dispersion was evaluated by conducting a series of core flooding experiments using multiple injection schemes.

\section{Experiment}

\subsection{Materials}

Na-AMPS nanogel was synthesized via a suspension polymerization process. 2-acrylamido 2-methyl propane sulfonic acid monomer (99\%), sodium dodecyl sulfate (SDS, > 99\%, $\mathrm{CMC}=2400 \mathrm{mg} / \mathrm{L}), \mathrm{Tween}^{\oplus} 60(\mathrm{CMC}=27 \mathrm{mg} / \mathrm{L}), N, N^{\prime}-$ methylene bis(acrylamide) (MBAA, 99\%), and sodium bicarbonate $\left(\mathrm{NHCO}_{3}, \geq 99.7 \%\right)$ were purchased from Sigma-Aldrich. Sorbitan monooleate $\left(\operatorname{Span}^{\odot} 80\right)$, sodium hydroxide ( $\mathrm{NaOH}, 97 \%)$, sodium chloride $(\mathrm{NaCl}, 99 \%)$, calcium chloride $\left(\mathrm{CaCl}_{2}\right.$, powder, $\left.97 \%\right)$, magnesium chloride $\left(\mathrm{MgCl}_{2}, 99 \%\right)$, and $n$-decane were purchased from Alfa Aesar. Ammonium persulfate $(\geq 98 \%)$ and sodium sulfate $\left(\mathrm{Na}_{2} \mathrm{SO}_{4}, \geq 99 \%\right)$ were purchased from Acros Organics. All chemicals were of reagent grade and used as received without further purification. Light mineral oil with a viscosity of $27.8 \mathrm{cP}$ (density $=0.833 \mathrm{~g} / \mathrm{mL}$ at $25^{\circ} \mathrm{C}$ ) was employed as the oil phase in all experiments. Due to its availability, seawater with a salinity that simulates the salinity of seawater in Saudi Arabia was used in all experiments unless otherwise mentioned. Seawater was prepared from deionized water and reagent-grade chemicals on the basis of a geochemical analysis reported by Yousef et al. (2011). The used seawater had a salinity of approximately $57,600 \mathrm{ppm}$ by weight. Table 1 lists the composition of the employed seawater. The density and viscosoity of the seawater are listed in Table 2. 
Table 1 Typical seawater composition in Saudi Arabia

\begin{tabular}{llllllll}
\hline Ion & Sodium & Calcium & Magnesium & Sulfate & Chloride & Bicarbonate & Total dissolved solids \\
\hline Concentration, ppm & 18,300 & 650 & 2110 & 4290 & 32,200 & 120 & 57,670 \\
\hline
\end{tabular}

Table 2 Density and viscosity of the employed seawater at room temperature of $25^{\circ} \mathrm{C}$

\begin{tabular}{ll}
\hline Property & Seawater \\
\hline Density, $\mathrm{g} / \mathrm{cm}^{3}$ & 1.040 \\
Viscosity, $\mathrm{cP}$ & 1.012 \\
\hline
\end{tabular}

\subsection{Nanogel synthesis}

Na-AMPS nanogel was prepared by a typical suspension polymerization process. The preparation process could be summarized as follows: $\mathrm{NaOH}$ is added to a stirred solution of 15 grams of 2-acrylamido 2-methyl propane sulfonic acid (AMPS) and 15 grams of deionized water at room temperature until the $\mathrm{pH}$ reaches exactly 7.0. Then, 0.1 gram of $N, N^{\prime}$ -methylene bis(acrylamide) (MBAA) is added to the solution while stirring. The solution is then added to $n$-decane (40 $\mathrm{mL})$ containing Span ${ }^{\odot} 80(21 \mathrm{~g})$ and Tween ${ }^{\odot} 60(9 \mathrm{~g})$ in a three-necked flask and bubbled with nitrogen, while it was kept in a water bath at $40{ }^{\circ} \mathrm{C}$ for $15 \mathrm{~min}$. After that, $0.2 \mathrm{~mL}$ of ammonium persulfate is added to the flask as an initiator. Stirring in the water bath is continued for 2 hours at $40^{\circ} \mathrm{C}$. Then, the emulsion is precipitated and washed with acetone and separated by centrifugation. The process of washing the emulsion with acetone is repeated several times to ensure that all surfactants and unreacted monomers are washed out.
The final isolated product is dried in the oven at $65^{\circ} \mathrm{C}$ for 24 h. Figure 1 shows samples of the dried and dispersed Na-AMPS nanogel.

\subsection{Preparation of nanogel and nanogel-surfactant solutions}

A stock solution of each nanogel and surfactant (SDS) was first prepared by dispersing the particles in seawater. Samples were prepared by diluting these stock solutions. Nanogel samples were prepared by incorporating nanogels at concentrations of $0.05,0.1,0.2,0.3,0.4,0.5,1.0$, and 1.5 wt $\%$. SDS was decided to be employed at a concentration of $0.1 \mathrm{wt} \%$ in all experiments (unless otherwise noted), which is lower than the critical micelle concentration (CMC) of SDS (2400 g/mL). CMC is defined as the surfactant concentration above which micelles will start to form. Higher concentrations of SDS have a slight effect (or even sometimes a negative effect) on enhancing oil recovery. For this reason, SDS was decided to have a concentration lower than CMC. In other words, the concentration of SDS was kept low to clearly visualize the effect of nanogel on oil recovery. Likewise, nanogel-surfactant dispersions were prepared by mixing different concentrations of nanogel and surfactant in seawater.

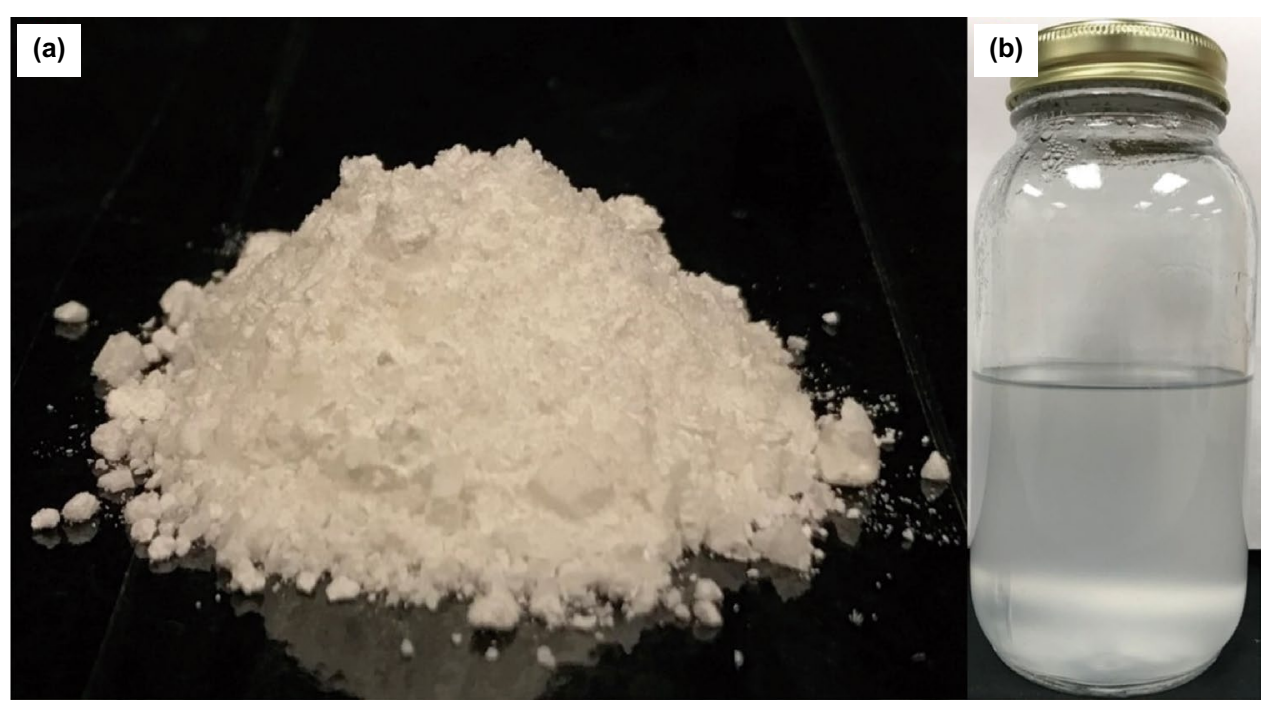

Fig. 1 a Dried Na-AMPS nanogel. b Na-AMPS nanogel dispersed in seawater 
Table 3 Petrophysical properties of core plugs

\begin{tabular}{lllllll}
\hline Core ID & Length, cm & Diameter, cm & Porosity, $\%$ & $\begin{array}{l}\text { Pore volume, } \\
\mathrm{cm}^{3}\end{array}$ & $\begin{array}{l}\text { Average liquid perme- } \\
\text { ability, } \mathrm{mD}\end{array}$ & $\begin{array}{l}S_{\text {wi }}, \% \\
S_{\text {oi }}, \%\end{array}$ \\
\hline A-1 & 12.65 & 5.08 & 19.5 & 50.4 & 134.3 & 40.4 \\
A-2 & 12.63 & 5.08 & 19.9 & 51.4 & 154.8 & 39.8 \\
A-3 & 12.59 & 5.08 & 19.2 & 50.4 & 134.7 & 63.2 \\
A-4 & 12.62 & 5.08 & 17.3 & 44.7 & 68.6 & 59.5 \\
A-5 & 12.67 & 5.08 & 18.4 & 47.5 & 74.2 & 37.4 \\
A-6 & 12.61 & 5.08 & 18.9 & 48.8 & 93.6 & 40.1 \\
A-7 & 12.57 & 5.08 & 19.7 & 50.9 & 122.9 & 59.9 \\
A-8 & 12.64 & 5.08 & 18.3 & 47.1 & 75.9 & 39.5 \\
A-9 & 12.50 & 5.08 & 18.6 & 48.0 & 84.3 & 39.7 \\
\hline
\end{tabular}

\subsection{Nanogel size distribution and zeta potential}

A nanosizer (Nano ZS, Malvern Instruments, UK) equipped with helium-neon laser $(633 \mathrm{~nm})$ was employed to determine the size distribution and obtain the zeta potential values of nanogel dispersions, both alone and combined with SDS. Dynamic light scattering (DLS) was used to measure the hydrodynamic radius of nanogel particles in the dispersing fluid. Furthermore, zeta potential values are essential for determining the charge nature of the particle surfaces. All measurements were taken at room temperature of $25^{\circ} \mathrm{C}$ and at a scattering angle of $90^{\circ}$. These measurements greatly help in studying the behavior of surfactant-nanogel systems and their molecular interactions.

\subsection{Rheological properties}

A Brookfield DV3T rheometer was employed to measure the rheological properties of seawater and the synthesized nanogel combined with SDS at $25^{\circ} \mathrm{C}$.

\subsection{Interfacial tension measurements}

Liquid-liquid interfacial tension between mineral oil and the aqueous nanogel dispersions and liquid-air surface tension between air and nanogel dispersions were measured using the pendant drop technique (ramè-hart advance goniometer $500-\mathrm{F} 1)$ for 1,000 seconds. The interfacial tension values were determined using the Young-Laplace equation. All measurements were taken under ambient conditions with a typical temperature of $25^{\circ} \mathrm{C}$.

\subsection{Porous media}

Several Berea sandstone (water-wet) core plugs with a low to medium permeability ranging from 68.6 to $154 \mathrm{mD}$ were employed in this study. The core plugs have a length of 5 inches $(12.6 \mathrm{~cm})$ and a diameter of 2 inches $(5.08 \mathrm{~cm})$. The porosity, liquid permeability, and pore volumes of the cores were determined using conventional core analysis methods. Table 3 summarizes the petrophysical properties of each core plug.

\subsection{Core flooding experiments}

Core plugs were mounted in a core holder that is designed for cores with 2 inches in diameter and up to $1 \mathrm{ft}$ in length. A schematic of the core flooding apparatus is shown in Fig. 2.

\subsubsection{Experimental procedure}

1. Core plugs are dried in an oven at $125^{\circ} \mathrm{C}$ for several weeks.

2. Core plugs are vacuumed for six hours and saturated with seawater.

3. Porosity and pore volumes are measured by weight difference and the density of the saturated brine at room temperature.

4. Core plugs are placed into a Hassler-type core holder and confined with a pressure of $850 \mathrm{psi}$ using a Teledyne ISCO model 500D syringe pump.

5. Absolute permeability is determined by injecting water at different flow rates.

6. Irreducible water saturation is established by injecting oil to displace water.

7. Initial water flooding is conducted at a flow rate of 0.5 $\mathrm{mL} / \mathrm{min}$ (which corresponds to a Darcy velocity of 1.16 $\mathrm{ft} /$ day until pressure stabilizes.

8. Different injection patterns of nanogel, surfactant, and post-water flooding were conducted into the core plugs at a flow rate of $0.5 \mathrm{~mL} / \mathrm{min}$. Only one PV of each NaAMPS nanogel and SDS was injected for most cases, while two PVs were injected for the nanogel-SDS oneslug injection cases. 


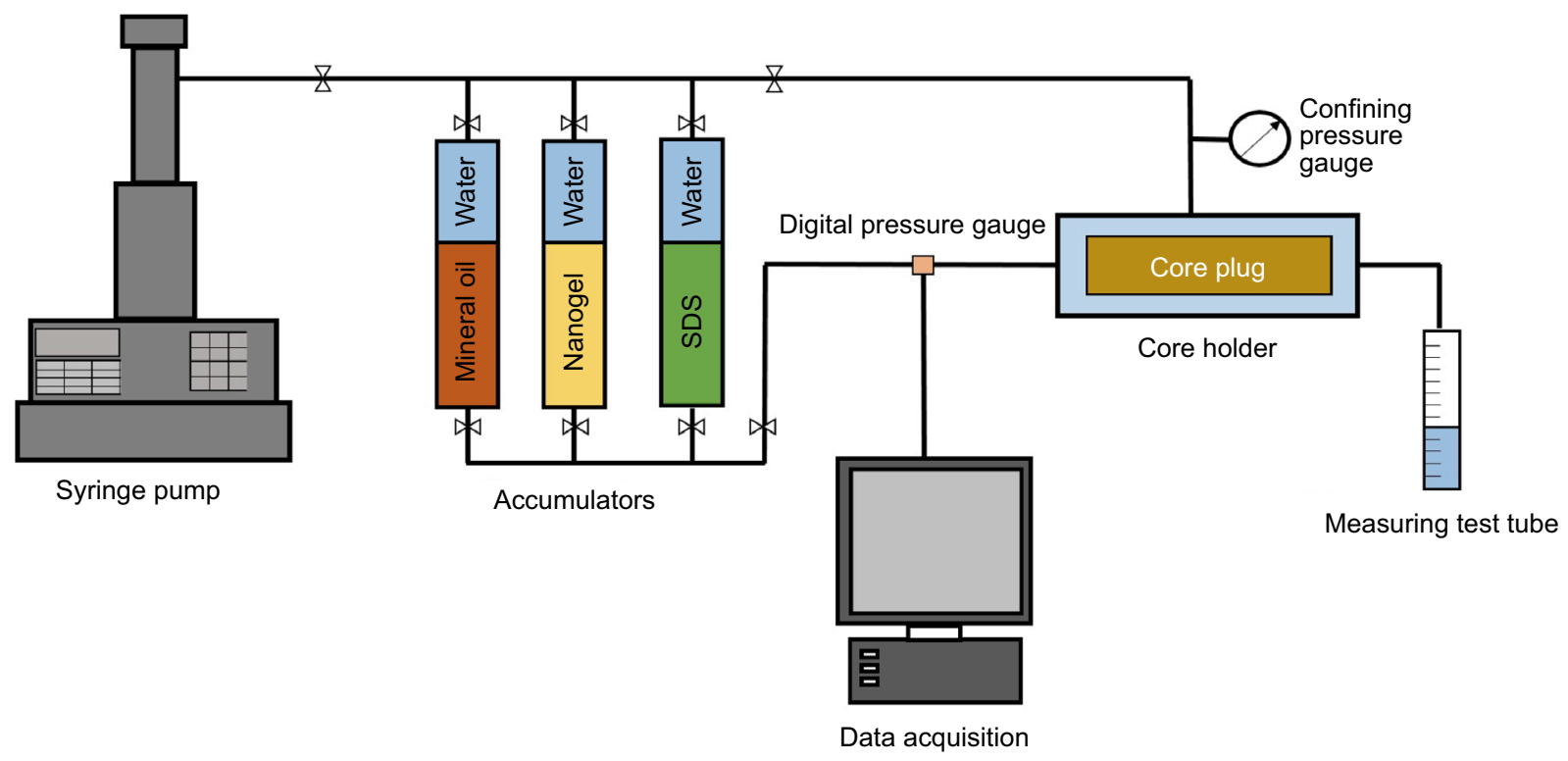

Fig. 2 Schematic of the experimental setup

The effluent samples that flowed through the core plugs were collected using measuring test tubes. Oil recovery was calculated using the amount of extracted oil from original oil in place. Additionally, a pressure transducer was installed at the inlet of the core holder to monitor the injection pressure. All core flooding experiments were conducted at a temperature of $25{ }^{\circ} \mathrm{C}$ to avoid the effects, if any, of nanogel thermal motion.

\subsubsection{Injection scheme}

In this work, three sets of flooding schemes have been conducted after seawater flooding. In the first set, enhanced recovery over nanogel flooding and SDS has been studied using one PV for each separate injection (sequential). For the second set, one PV of Na-AMPS nanogel was injected after initial seawater flooding, and one PV of SDS was injected after the post-seawater flooding (sequential). In the last set, two PVs of Na-AMPS nanogel and SDS as one slug were injected after initial seawater flooding (one-slug). Table 4 summarizes the injection schedules for each employed core along with the studied parameter.

\section{Results and discussion}

\subsection{Size distribution of nanogel}

Table 5 illustrates the physiochemical properties of the synthesized Na-AMPS nanogel $(0.1 \mathrm{wt} \%)$, including the surface $\zeta$-potential, $\mathrm{pH}$ and polydispersity index (PDI) in seawater. The average hydrodynamic diameter of the nanogel in seawater is $222.5 \mathrm{~nm}$, as shown in Fig. 3. The size of nanogel is affected by the salinity of the displacing fluid. This was confirmed by measuring the size distribution of the nanogel in diluted seawater $(0.58 \mathrm{wt} \%)$. It was observed that the diameter of the nanogel expanded in diluted seawater to $242.2 \mathrm{~nm}$, as demonstrated in Fig. 3. This figure also shows that both size distribution curves exhibited a mono model distribution, with one peak pointing to a predominant homogeneous droplet size. Nanogel dispersed in seawater shows a good stability during a period of two weeks by well maintaining the structural size within $220-225 \mathrm{~nm}$, as shown in Fig. 4. Furthermore, the stability of nanogel dispersions is suggested by zeta potential measurements. Increasing trend of zeta potential refers to better stability for the dispersion. Here, the magnitude of the zeta potential measurements shows an increasing trend with concentrated nanogels, as illustrated in Fig. 5. It is possible that this increase in the magnitude is due to the increase in the total number of charged particles in the concentrated solutions or the charge increase per particle.

\subsection{Rheological properties}

As expected, the viscosity of nanogel dispersions (with and without the addition of SDS surfactant) was affected by the concentration of nanogels. As shown in Fig. 6, the viscosity of nanogel dispersions was almost constant at low shear rates $\left(<7 \mathrm{~s}^{-1}\right)$, which suggests Newtonian behavior. However, the viscosity gradually increases at higher shear rates, which suggests shear thickening behavior. The viscosity at low shear rates was Newtonian because the formation of the interparticle structure was hindered by the electrostatic 
Table 4 Injection schedules for each core used in the experiments

\begin{tabular}{|c|c|c|c|c|c|c|}
\hline \multirow[t]{2}{*}{ Core ID } & \multirow[t]{2}{*}{ Injection mode } & \multirow[t]{2}{*}{ Purpose } & \multicolumn{4}{|c|}{ Injection schedules } \\
\hline & & & 1 & 2 & 3 & 4 \\
\hline A-1 & - & Base case & SW & NG & SW & - \\
\hline A-2 & Sequential & NG-SDS sequence effect & SW & NG & SW & SDS \\
\hline A-3 & Sequential & NG-SDS sequence effect & SW & NG & SDS & SW \\
\hline A-4 & Sequential & NG concentration effect & SW & $2 * \mathrm{NG}^{\mathrm{a}}$ & SDS & SW \\
\hline A-5 & Sequential & NG concentration effect & SW & $3 * \mathrm{NG}$ & SDS & SW \\
\hline A-6 & Sequential & NG concentration effect & SW & $5 * \mathrm{NG}$ & SDS & SW \\
\hline A-7 & Sequential & NG concentration effect & SW & $10 * \mathrm{NG}$ & SDS & SW \\
\hline A-8 & One-slug & Diluted nanogel effect & SW & $0.5^{*}(\mathrm{NG}+\mathrm{SDS})$ & SW & $\mathrm{DSW}^{\mathrm{b}}$ \\
\hline A-9 & One-slug & NG concentration effect & SW & NG+SDS & SW & - \\
\hline
\end{tabular}

a 2 PV of nanogel

${ }^{\mathrm{b}} 10$-times diluted seawater

Table 5 Physiochemical properties of the synthesized nanogel with a concentration of $0.1 \mathrm{wt} \%$ dispersed in seawater

\begin{tabular}{llll}
\hline Charge & Surface $\zeta$-potential, $\mathrm{mV}$ & Polydispersity index (PDI) & $\mathrm{pH}$ \\
\hline Negative & -21.5 & 0.215 & 7.0
\end{tabular}

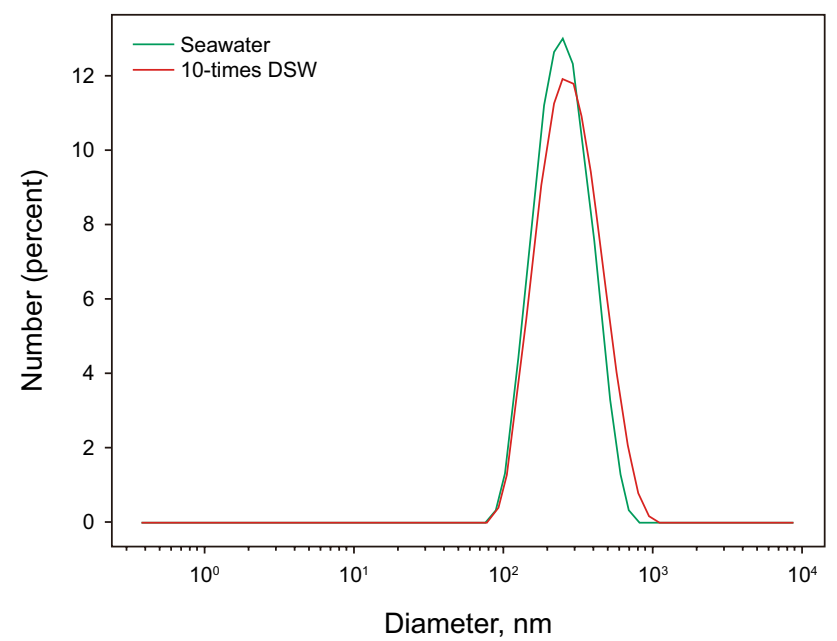

Fig. 3 Hydrodynamic diameter distribution of Na-AMPS nanogel in seawater and diluted seawater measured at a concentration of $1 \mathrm{~g} / \mathrm{L}$ and a temperature of $25^{\circ} \mathrm{C}$

repulsion. On the other hand, when the shear rate is above $120 \mathrm{~s}^{-1}$, the attraction of nanogel dispersions was increased, which caused the viscosity to gradually rise. Moreover, as the shear rate increases, nanogel dispersions experience a sudden increase in viscosity measurements, which might be caused by the increased interaction among particles due to high rotational speed. Nevertheless, a higher concentration

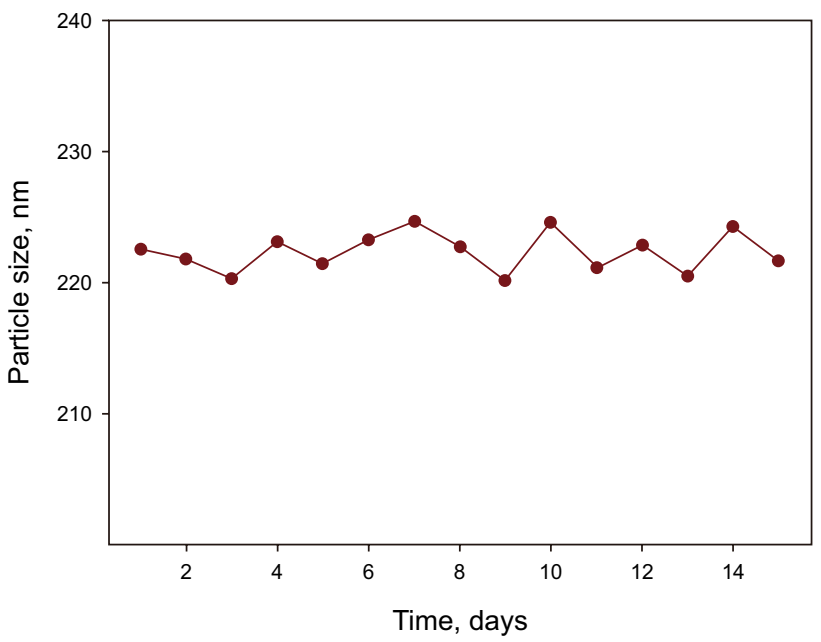

Fig. 4 Nanogel stability evaluation in seawater for a two-week time period

of nanogel dispersion (1.5 wt\%) did not follow this trend. It was observed that higher-concentration dispersions experienced shear thinning behavior with increasing shear rate, as shown in Fig. 6. This could be attributed to the change in the microstructure of nanogel dispersions at higher shear rates (Wagner and Brady 2009).

Furthermore, the relative viscosity $\eta_{\mathrm{r}}$ is defined as the ratio of the viscosity of nanogel combined with SDS solution to the viscosity of the nanogel only solution at a constant shear rate. The relative viscosities $\eta_{r}$ of nanogel dispersions combined with SDS at a constant shear rate of 120 $\mathrm{s}^{-1}$ at different concentrations are presented in Fig. 7. It was found that the relative viscosity $\eta_{\mathrm{r}}$ is fitted by an exponential function of nanogel concentration. The change in slope indicates a change in polymer interaction in nanogel dispersions (Gupta et al. 2005). When the concentration of nanogel 


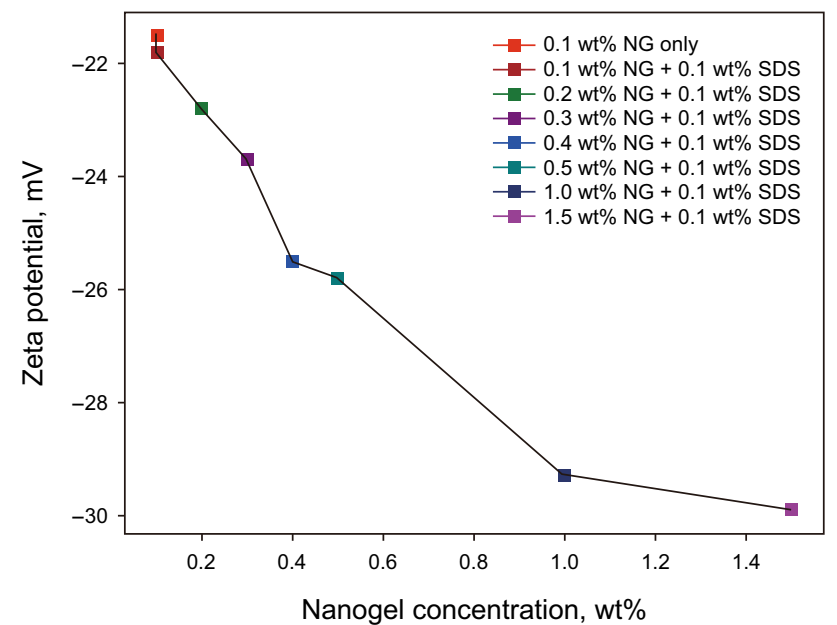

Fig. 5 Change in zeta potential in the mixed nanogel-SDS solutions dispersed in seawater and measured at $25{ }^{\circ} \mathrm{C}$. SDS concentration is kept constant at $0.1 \mathrm{wt} \%$

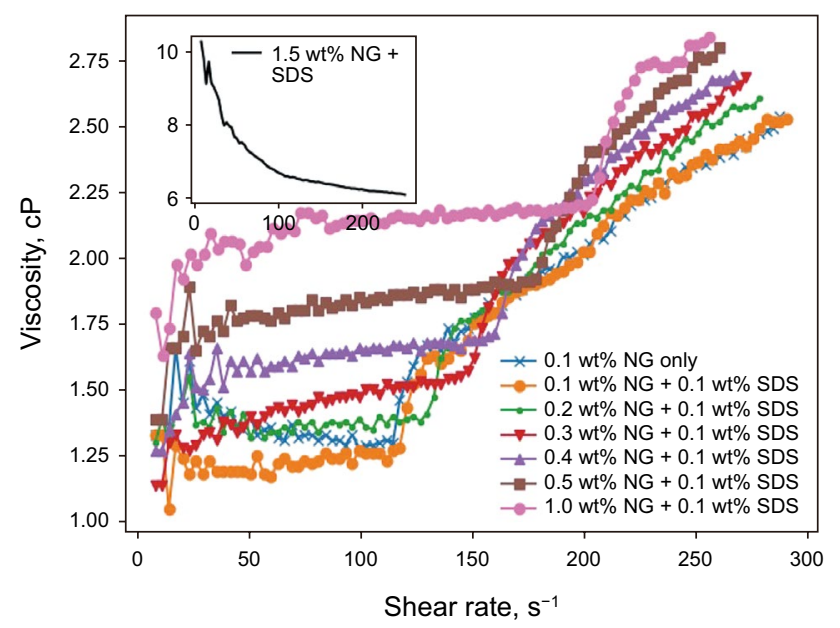

Fig. 6 Viscosity of varying concentration of nanogel dispersions combined with SDS at different shear rates. SDS concentration is kept constant at $0.1 \mathrm{wt} \%$

dispersion is below $0.3 \mathrm{wt} \%$, the nanogel is fully dispersed in the displacing fluid and the interactions between them fully dominate the flow behavior of nanogel dispersions. On the other hand, when the concentration of nanogel is above $0.4 \mathrm{wt} \%$, the spacing between particles is greatly reduced and the interactions between neighboring particles are no longer minimal.

\subsection{Interfacial tension measurements}

To understand the mechanism of nanogel flooding, IFT measurements were performed on nanogel-air and nanogel-mineral oil using the pendant drop method. Table 6 lists

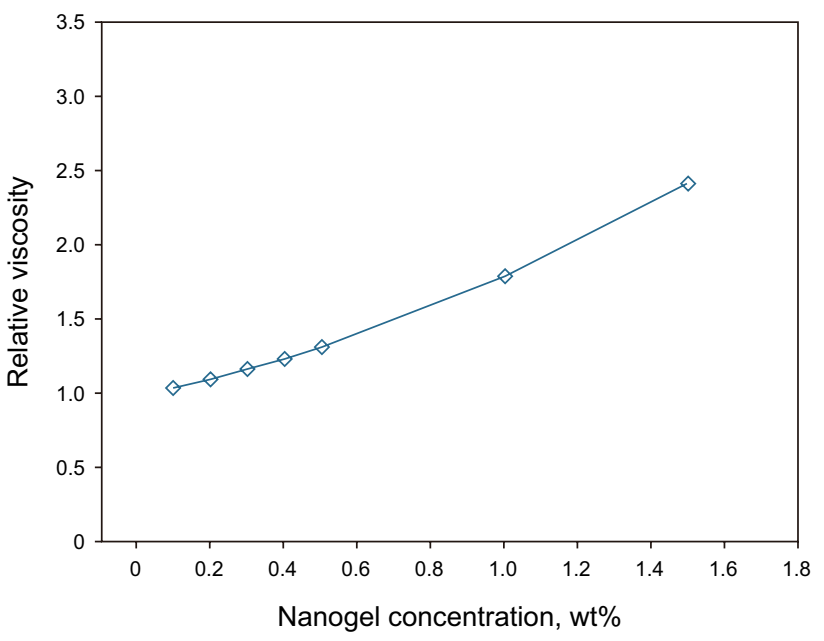

Fig. 7 The relative viscosity $\eta_{\mathrm{r}}$ is the ratio of the viscosity of nanogel combined with SDS solution to the viscosity of the nanogel only solution at a constant shear rate. The blue curve represents the relative viscosity $\eta_{\mathrm{r}}$ of different concentrations of nanogel dispersions combined with SDS in seawater at a shear rate of $120 \mathrm{~s}^{-1}$. SDS concentration is kept constant at $0.1 \mathrm{wt} \%$.

Table 6 Surface and interfacial tension measurements of nanogel dispersions

\begin{tabular}{|c|c|c|}
\hline Type & $\begin{array}{l}\text { Liquid-air } \\
\text { surface tension, } \\
\mathrm{mN} / \mathrm{m}\end{array}$ & $\begin{array}{l}\text { Liquid-liquid } \\
\text { interfacial tension, } \\
\mathrm{mN} / \mathrm{m}\end{array}$ \\
\hline Mineral Oil & 28.72 & - \\
\hline Seawater & 71.47 & 34.36 \\
\hline $0.1 \mathrm{wt} \%$ nanogel & 45.88 & 26.52 \\
\hline $\begin{array}{l}0.1 w t \% \text { nanogel }+0.1 \text { wt } \% \\
\text { SDS }\end{array}$ & 17.54 & 23.39 \\
\hline $\begin{array}{l}0.2 \mathrm{wt} \% \text { nanogel }+0.1 \mathrm{wt} \% \\
\quad \text { SDS }\end{array}$ & 16.85 & 15.80 \\
\hline $\begin{array}{l}0.3 w t \% \text { nanogel }+0.1 \text { wt } \% \\
\text { SDS }\end{array}$ & 15.13 & 11.04 \\
\hline $\begin{array}{l}0.4 w t \% \text { nanogel }+0.1 w t \% \\
\text { SDS }\end{array}$ & 12.57 & 8.22 \\
\hline $\begin{array}{l}0.5 w t \% \text { nanogel }+0.1 w t \% \\
\text { SDS }\end{array}$ & 10.27 & 6.91 \\
\hline $\begin{array}{l}1.0 \mathrm{wt} \% \text { nanogel }+0.1 \mathrm{wt} \% \\
\text { SDS }\end{array}$ & 2.51 & NA \\
\hline
\end{tabular}

the results of surface and interfacial tension measurements. It is evident from this table and Figs. 8 and 9 that the addition of SDS surfactant to Na-AMPS nanogel dispersions considerably reduces the interfacial tension between both nanogel-air and nanogel-oil phase. A number of studies have shown that particles with appropriate surface charge stabilize emulsion droplets by the formation of a twodimensional close-packed structure on the oil-water interface (Eskandar et al. 2011; Binks et al. 2003). Furthermore, the high tendency of tested nanogel dispersions to adsorb at 


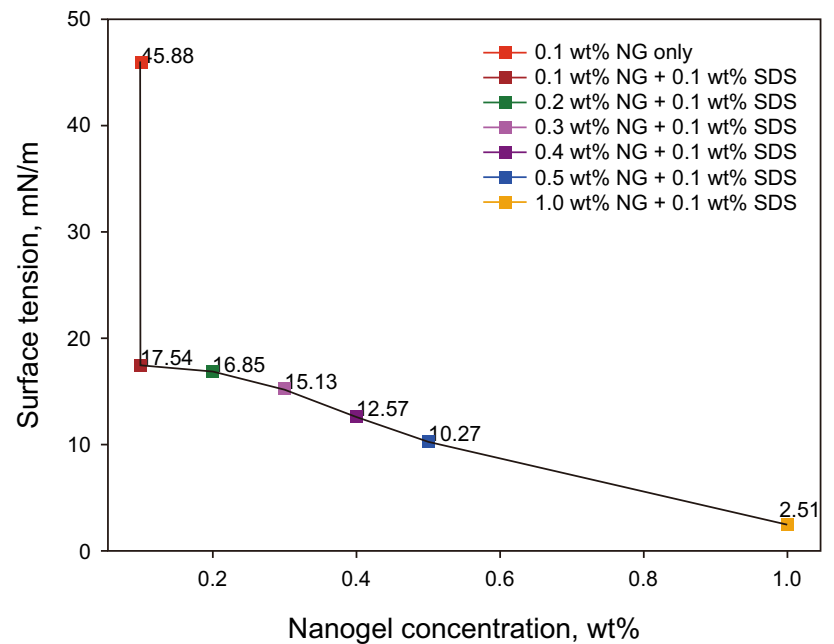

Fig. 8 Surface tension between air and varying concentration of nanogel dispersions combined with SDS at $25^{\circ} \mathrm{C}$. SDS concentration is kept constant at $0.1 \mathrm{wt} \%$

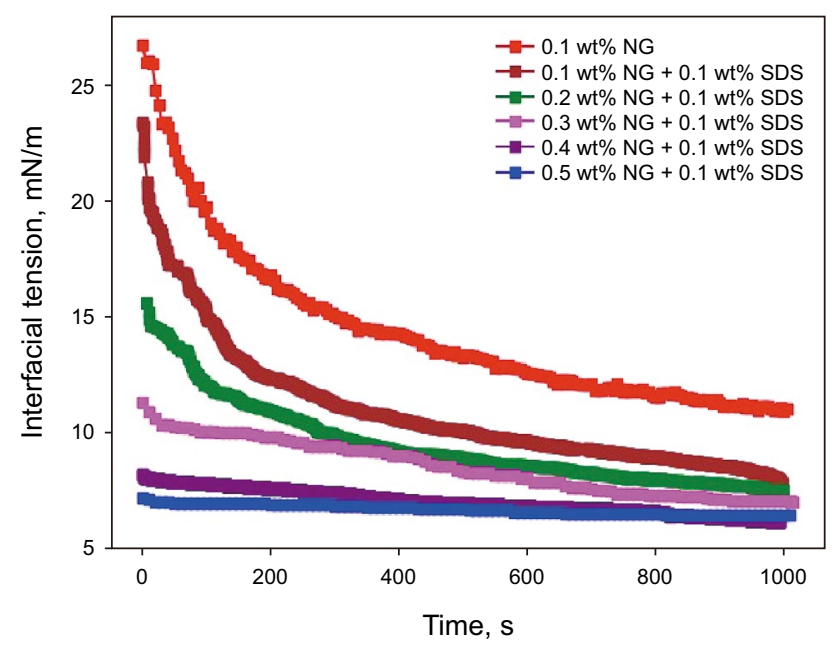

Fig. 9 Dynamic interfacial tension between mineral oil and varying concentrations of nanogel dispersions combined with SDS. SDS concentration is kept constant at $0.1 \mathrm{wt} \%$

liquid-liquid interfaces is attributed to the effect of dissolved oil in the water phase.

Figure 9 shows the liquid-liquid interfacial tensions between oil phase and nanogel dispersions in the presence of both Na-AMPS nanogel and SDS. The figure demonstrates the effect of surfactant and particle concentrations on the interfacial tension measurements and indicates that in diluted surfactant and concentrated nanogels, the interfacial tension values are lower than that of the basic Na-AMPS solution $(0.1 \mathrm{wt} \%)$. Thus, the addition of a small amount of SDS $(0.1 \mathrm{wt} \%)$ to Na-AMPS nanogel solution greatly reduces the interfacial tension values. In other words, the particle adsorption energy reduces further to more negative

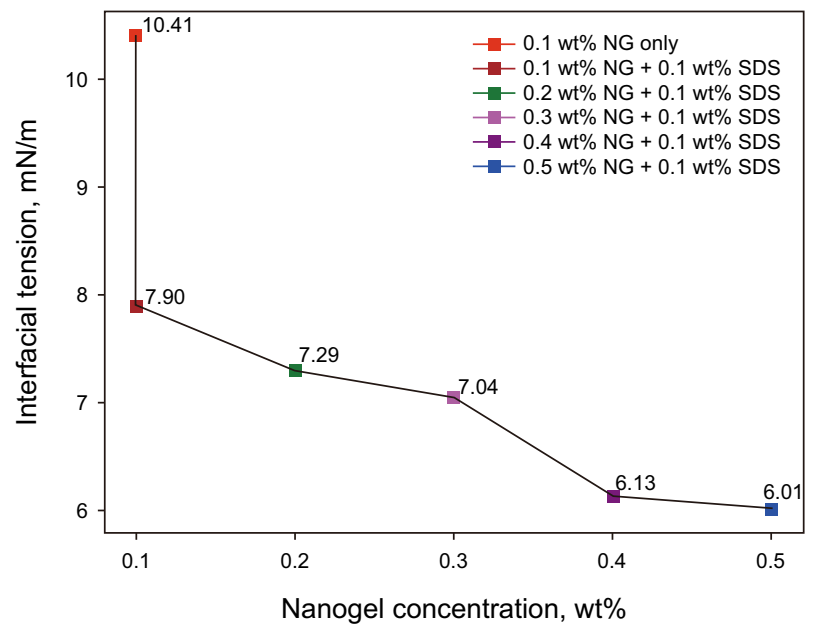

Fig. 10 Equilibrium interfacial tension of nanogel dispersions combined with SDS. SDS concentration is kept constant at $0.1 \mathrm{wt} \%$

values in the presence of SDS. It is worth mentioning that the addition of nanogel has no effect on the interfacial tension values when SDS concentration is equal or above the critical micelle concentration (CMC) (Jiang et al. 2016). Our results of the effect of SDS on interfacial tension values of nanogel dispersions are consistent with the findings of Dong et al. (2010); and Geng et al. (2018b). Similar results were obtained for air-liquid surface tension values in the presence of Na-AMPS nanogel and SDS, as presented in Fig. 8. The adsorption energy of particles to the liquid-air interface is lower than that of liquid-liquid interface. This explains the relatively higher surface tension values between nanogel dispersions and air. In addition, as Figs. 9 and 10 illustrate, nanogel dispersions with a concentration below $0.2 \mathrm{wt} \%$ reached the equilibrium interfacial tension, where the adsorption rate of nanogel onto oil-water interface is equal to the desorption rate, in about 1,000 seconds. However, for nanogel dispersions with higher concentrations, the equilibrium state was reached much faster.

\subsection{Confirmation of enhanced oil recovery by core flooding}

Series of core flooding experiments were conducted with water floods and injection of different concentrations of Na-AMPS nanogel and a fixed concentration of SDS surfactant $(0.1 \mathrm{wt} \%)$ as one-slug injection and separate injections in Berea sandstone core plugs. Experimentally, it is confirmed that the oil recovery using Na-AMPS nanogel and SDS was higher than seawater flooding only. The incremental oil recovery by nanogel injection greatly increases with the concentration of the nanogel. The initial oil recovery by seawater flooding was $43.3 \%$, compared to $48.6 \%$ with $0.1 \mathrm{wt} \%$ of Na-AMPS nanogel injection, and $55.7 \%$ with 


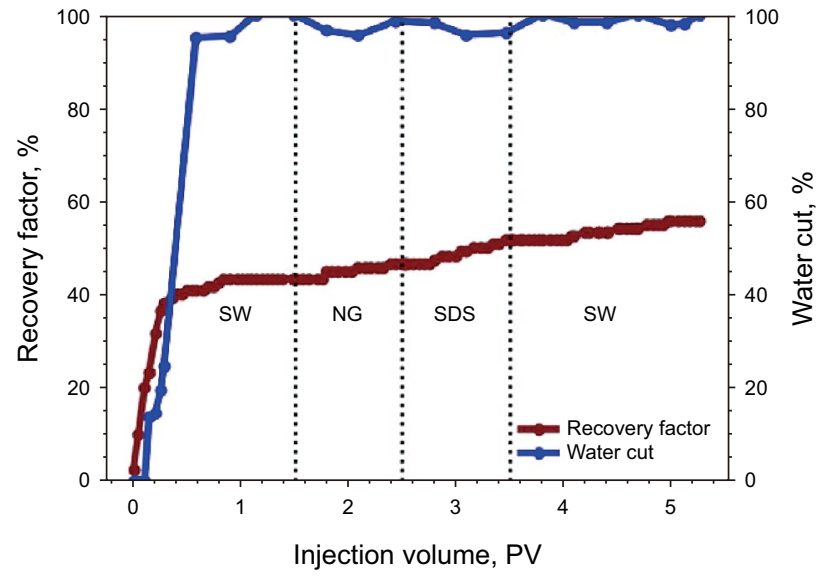

Fig. 11 Oil recovery factor and water cut results of core A-3 using sequential injections of nanogel and SDS followed by seawater flooding. The brown curve represents the amount of oil produced in terms of OOIC through all injection slugs. The blue curve represents the water cut through all injection slugs

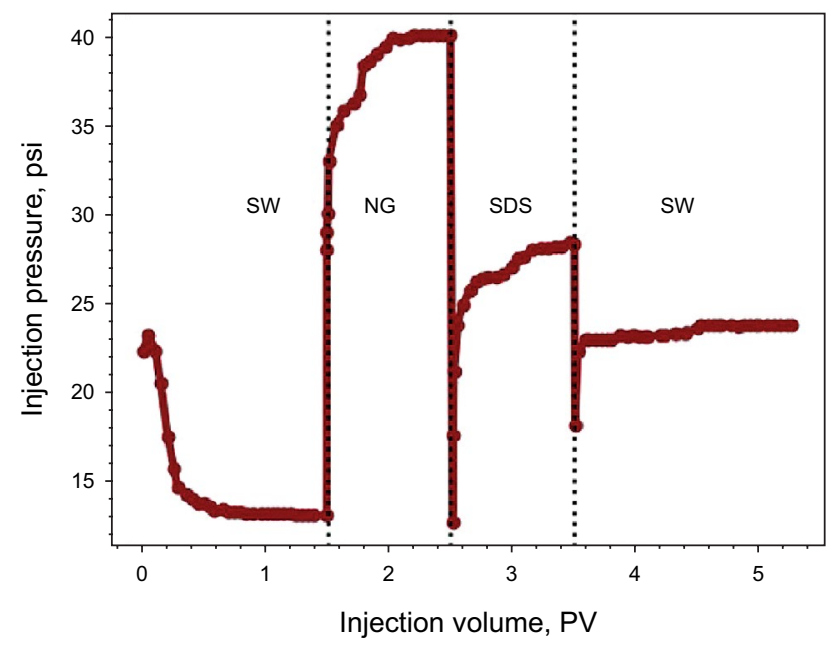

Fig. 12 Injection pressure results of core A-3 using sequential injections of nanogel and SDS followed by seawater flooding

$0.1 \mathrm{wt} \%$ of Na-AMPS nanogel followed by $0.1 \mathrm{wt} \%$ SDS injection, as illustrated in Fig. 11. Furthermore, the trend of injection pressure for both Na-AMPS nanogel and SDS was almost identical, as both stabilized toward the end of their segment injection, especially in low nanogel concentrations, as shown in Fig. 12. All core flooding results are given in the Appendix. Resistance factor and residual resistance factor are two terms used to evaluate the injectivity process and plugging efficiency of gel treatments. Resistance factor $\left(F_{\mathrm{r}}\right)$ is defined as the ratio between water mobility $\left(\lambda_{\mathrm{w}}\right)$ and nanogel mobility $\left(\lambda_{\text {ng }}\right)$. In other words, it is the ratio of pressure drop across the core caused by the injection of nanogel dispersion $\left(\Delta P_{\mathrm{ng}}\right)$ to the pressure drop caused by the injection of brine $\left(\Delta P_{\mathrm{w}}\right)$ at the same flow rate (Eq. 1).

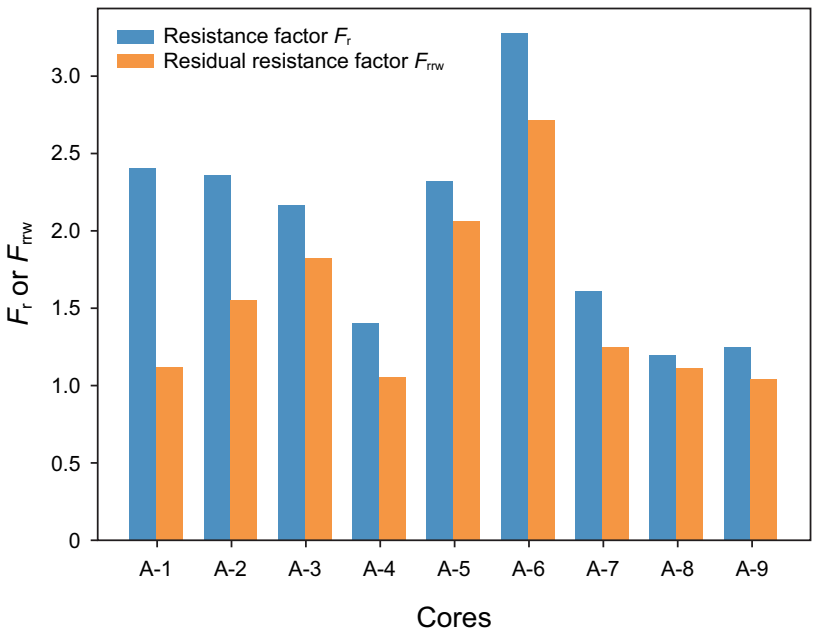

Fig. 13 Resistance factor calculated at the end of NG/SDS injection and residual resistance factor calculated using stabilized pressure of last water slug of all employed cores

Residual resistance factor to water $\left(F_{\text {rrw }}\right)$ is the ratio between water mobility before and after nanogel treatment (Eq. 2). As shown in Fig. 13, nanogels are not recommended for use as strong plugging materials in sandstone reservoirs with permeability higher than $100 \mathrm{mD}$ (plugging efficiency in this case was $40 \%$ ). The figure also shows that the injectivity of nanogel was not much higher than that of seawater. In this case, $F_{\mathrm{r}}$ and $F_{\text {rrw }}$ were 2.1 and 1.7 , respectively.

$F_{\mathrm{r}}=\frac{\lambda_{\mathrm{w}}}{\lambda_{\mathrm{ng}}}=\frac{\Delta P_{\mathrm{ng}}}{\Delta P_{\mathrm{w}}}$

$F_{\text {rrw }}=\frac{\left(\lambda_{\mathrm{w}}\right)_{\text {Before }}}{\left(\lambda_{\mathrm{w}}\right)_{\text {After }}}=\frac{\left(\Delta P_{\mathrm{w}}\right)_{\text {After }}}{\left(\Delta P_{\mathrm{w}}\right)_{\text {Before }}}$

A thorough investigation of the interfacial tension between different concentrations of nanogel dispersions and the oil phase can possibly explain the enhancement in oil recovery. The equilibrium interfacial tension between $0.1 \mathrm{wt} \%$ nanogel and oil phase was measured to be $10.41 \mathrm{mN} / \mathrm{m}$. Upon adding $0.1 \mathrm{wt} \%$ SDS to the nanogel dispersion, the equilibrium interfacial tension value was reduced to $7.90 \mathrm{mN} / \mathrm{m}$, as shown in Fig. 10. A similar trend was observed for higher concentrations of Na-AMPS nanogel with $0.1 \mathrm{wt} \%$ SDS, as illustrated in Table 6.

Additionally, the effect of injecting diluted seawater was studied during core flooding experiments. As shown in Fig. 14, it is observed that the injection pressure of the diluted seawater was higher than that of the concentrated seawater. This can be due to the fact that nanogels reacted to diluted seawater by further swelling and expanding in their diameters. 


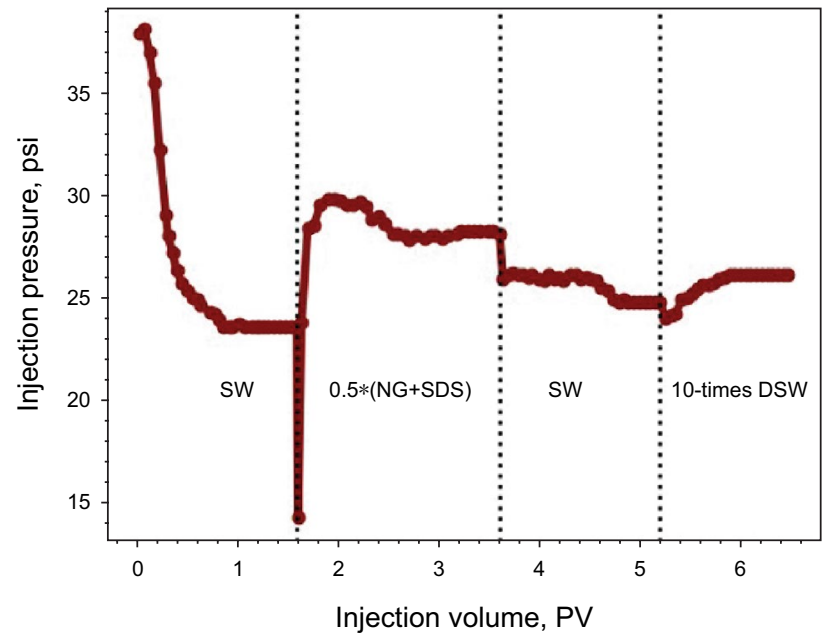

Fig. 14 Injection pressure results of core A-8 using one-slug injection of nanogel-SDS (0.05 wt\% each) followed by alternating seawater and 10 times diluted seawater injections

The recovered oil from core flooding tests varied with different injection schemes. Table 7 summarizes core flooding experiments when one PV of each Na-AMPS nanogel and SDS was injected and followed by extended seawater flooding. This injection scheme recovered higher amounts of oil compared to the other schemes. Figures 11 and 15 demonstrate that injecting Na-AMPS nanogel and SDS before the post-seawater flooding resulted in higher oil recovery by a factor of $2.6 \%$ when compared to injecting SDS after post-seawater flooding. Table 8 compares between separate and one-slug injection schemes and illustrates that separate injections of Na-AMPS nanogel and SDS recovered higher amounts of oil when compared to one-slug injection with the same concentration. Although one-slug injection is a common practice in field operations, laboratory-scale experiments showed that this injection scheme might not be the optimum.

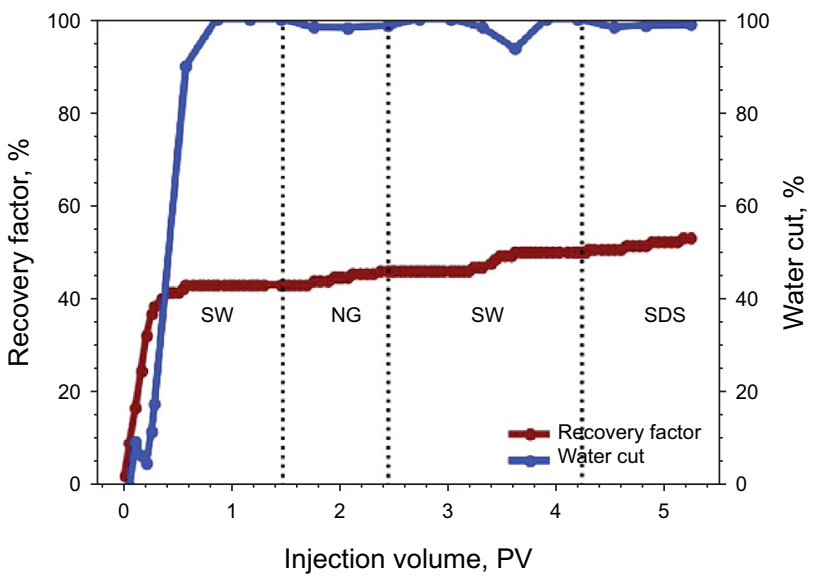

Fig. 15 Oil recovery factor and water cut results of core A-2 using sequential injections of nanogel, seawater and SDS. The brown curve represents the amount of oil produced in terms of OOIC through all injection slugs. The blue curve represents the water cut through all injection slugs

\section{Conclusions}

The behavior and transportation of polymeric cross-linked nanogels are attracting more attention due to their stability in water with high salinity and ability to extract higher amounts of oil by adsorbing at the oil-water interface. In this work, negatively charged Na-AMPS nanogel and SDS surfactant were employed as potential feasible materials for enhanced oil recovery in sandstone reservoirs. The main results that could be obtained from this study are summarized as follows:

1. Na-AMPS nanogel can be dispersed in high-salinity waters and still form a stable solution. This could be observed from the narrow size distribution with one

Table 7 Summary of sequential injection core flooding experiments

\begin{tabular}{|c|c|c|c|c|c|c|}
\hline Core ID & Scenario & $\begin{array}{l}\text { Seawater flooding } \\
\text { recovery, } \%\end{array}$ & $\begin{array}{l}\text { Nanogel recov- } \\
\text { ery, } \%\end{array}$ & SDS recovery, $\%$ & $\begin{array}{l}\text { Post-seawater flood- } \\
\text { ing recovery, } \%\end{array}$ & Total, \% \\
\hline A-1 & $0.1 \mathrm{wt} \% \mathrm{NG}$ & 43.3 & 2.8 & - & 2.5 & 48.6 \\
\hline A-3 & $0.1 \mathrm{wt} \% \mathrm{NG}+0.1 \mathrm{wt} \% \mathrm{SDS}$ & 43.2 & 3.0 & 5.3 & 4.2 & 55.7 \\
\hline A-4 & $0.2 \mathrm{wt} \% \mathrm{NG}+0.1 \mathrm{wt} \% \mathrm{SDS}$ & 43.1 & 4.5 & 6.3 & 4.5 & 58.4 \\
\hline A-5 & $0.3 \mathrm{wt} \% \mathrm{NG}+0.1 \mathrm{wt} \% \mathrm{SDS}$ & 43.4 & 6.1 & 6.1 & 3.5 & 59.1 \\
\hline A-6 & $0.5 \mathrm{wt} \% \mathrm{NG}+0.1 \mathrm{wt} \% \mathrm{SDS}$ & 43.2 & 6.4 & 4.3 & 3.4 & 57.3 \\
\hline A-7 & $1.0 \mathrm{wt} \% \mathrm{NG}+0.1 \mathrm{wt} \% \mathrm{SDS}$ & 43.1 & 8.1 & 4.1 & 2.4 & 57.7 \\
\hline
\end{tabular}


Table 8 Comparison between sequential and one-slug injection schemes

\begin{tabular}{|c|c|c|c|c|c|c|c|}
\hline Core ID & Scenario & Mode & $\begin{array}{l}\text { Seawater flood- } \\
\text { ing recovery, \% }\end{array}$ & $\begin{array}{l}\text { Nanogel } \\
\text { recovery, } \\
\%\end{array}$ & $\begin{array}{l}\text { SDS recovery, } \\
\%\end{array}$ & $\begin{array}{l}\text { Post-seawater } \\
\text { flooding recov- } \\
\text { ery, } \%\end{array}$ & $\begin{array}{l}\text { Total, } \\
\%\end{array}$ \\
\hline A-2 & $0.1 \mathrm{wt} \% \mathrm{NG}+0.1 \mathrm{wt} \% \mathrm{SDS}$ & Separate injections & 43.1 & 3.1 & 3.1 & 3.8 & 53.1 \\
\hline A-3 & $0.1 \mathrm{wt} \% \mathrm{NG}+0.1 \mathrm{wt} \% \mathrm{SDS}$ & Separate injections & 43.2 & 3.0 & 5.3 & 4.2 & 55.7 \\
\hline A-8 & $0.05 \mathrm{wt} \% \mathrm{NG}+0.05 \mathrm{wt} \% \mathrm{SDS}$ & One-slug injection & 43.1 & 4.3 & & 3.5 & 50.9 \\
\hline A-9 & $0.1 \mathrm{wt} \% \mathrm{NG}+0.1 \mathrm{wt} \% \mathrm{SDS}$ & One-slug injection & 43.3 & 4.5 & & 2.5 & 50.3 \\
\hline
\end{tabular}

Injection schedule of this core is $\mathrm{NG} \rightarrow \mathrm{SW} \rightarrow \mathrm{SDS}$

peak pointing to a predominant homogeneous droplet size when dispersed in seawater.

2. Nanogel dispersion shows good long-term stability during a period of two weeks. The structural size of nanogel in seawater was well maintained for this period with a hydrodynamic diameter within 220-225 $\mathrm{nm}$.

3. The viscosity of nanogel-SDS dispersions was affected by the concentration of nanogels. Lower concentrations of nanogels showed shear thickening behavior at higher shear rates, while higher nanogel concentrations show shear thinning behavior.

4. The interfacial tension (IFT) reduced when low concentration of SDS was introduced to Na-AMPS nanogel dispersions. Lower IFT values were observed with increasing nanogel concentration from $0.1 \mathrm{wt} \%$ to 1.0 wt $\%$. This implies that IFT reduction might be a major EOR mechanism in nanogel-surfactant flooding.

5. Injection schemes of nanogel and surfactant played an essential role in the amount of recovered oil in sandstone core plugs. The results showed that sequential injections of nanogel and SDS after initial seawater flooding is the better scheme among the ones tested.

6. The results of core flooding experiments confirm that Na-AMPS nanogel combined with SDS could improve the oil recovery factor by $15 \%$ after initial seawater flooding.

Open Access This article is licensed under a Creative Commons Attribution 4.0 International License, which permits use, sharing, adaptation, distribution and reproduction in any medium or format, as long as you give appropriate credit to the original author(s) and the source, provide a link to the Creative Commons licence, and indicate if changes were made. The images or other third party material in this article are included in the article's Creative Commons licence, unless indicated otherwise in a credit line to the material. If material is not included in the article's Creative Commons licence and your intended use is not permitted by statutory regulation or exceeds the permitted use, you will need to obtain permission directly from the copyright holder. To view a copy of this licence, visit http://creativecommons.org/licenses/by/4.0/.

\section{Appendix}

See Figs. 16, 17, 18, 19, 20, 21, 22 and 23.

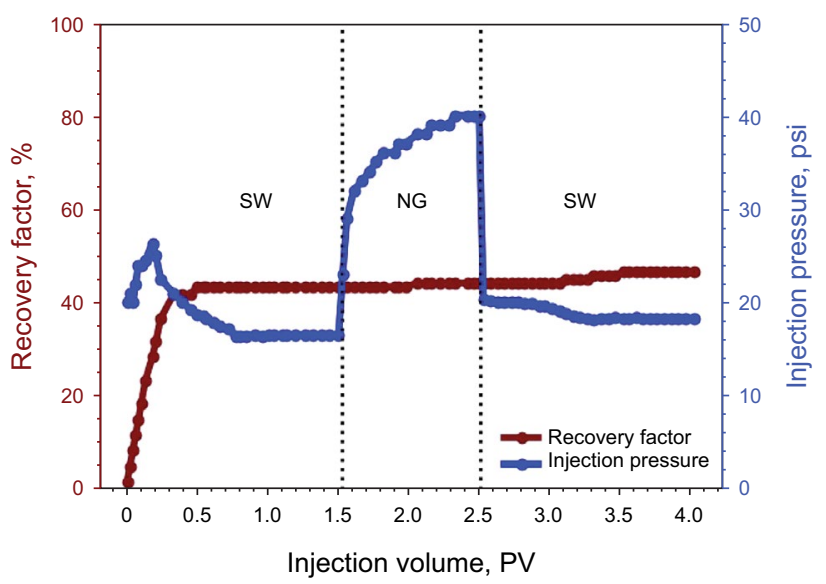

Fig. 16 Oil recovery factor and injection pressure results of core A-1 (base case) using nanogel injection followed by seawater flooding

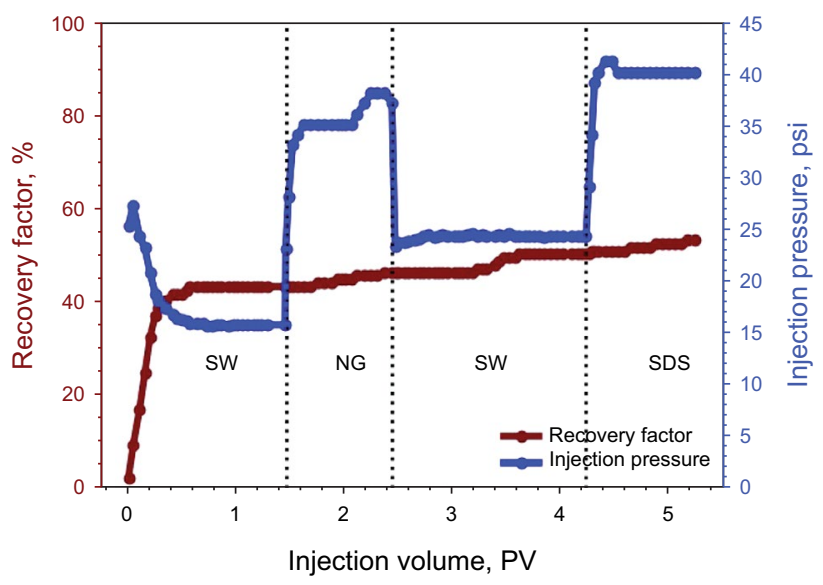

Fig. 17 Oil recovery factor and injection pressure results of core A-2 using nanogel injection followed by seawater flooding and SDS injection 


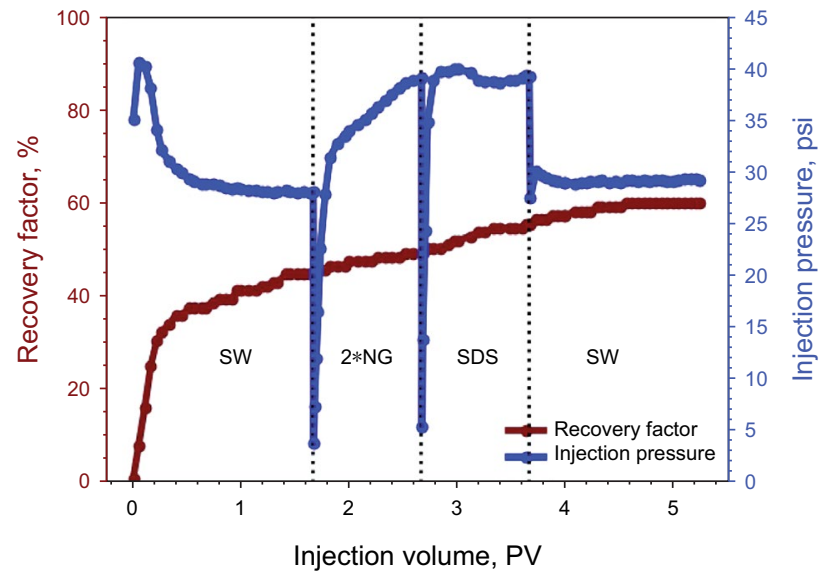

Fig. 18 Oil recovery factor and injection pressure results of core A-4 using sequential injections of $0.2 \mathrm{wt} \%$ nanogel and $0.1 \mathrm{wt} \%$ SDS followed by seawater flooding

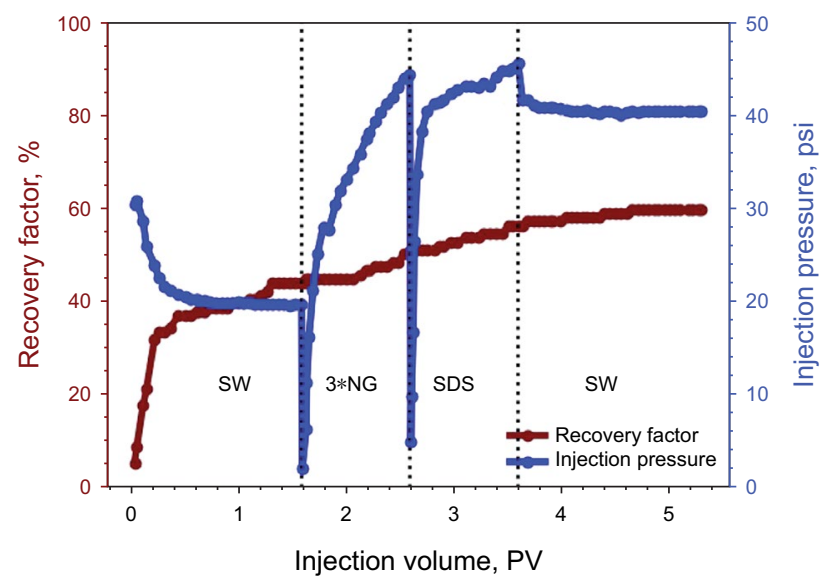

Fig. 19 Oil recovery factor and injection pressure results of core A-5 using sequential injections of $0.3 \mathrm{wt} \%$ nanogel and $0.1 \mathrm{wt} \%$ SDS followed by seawater flooding

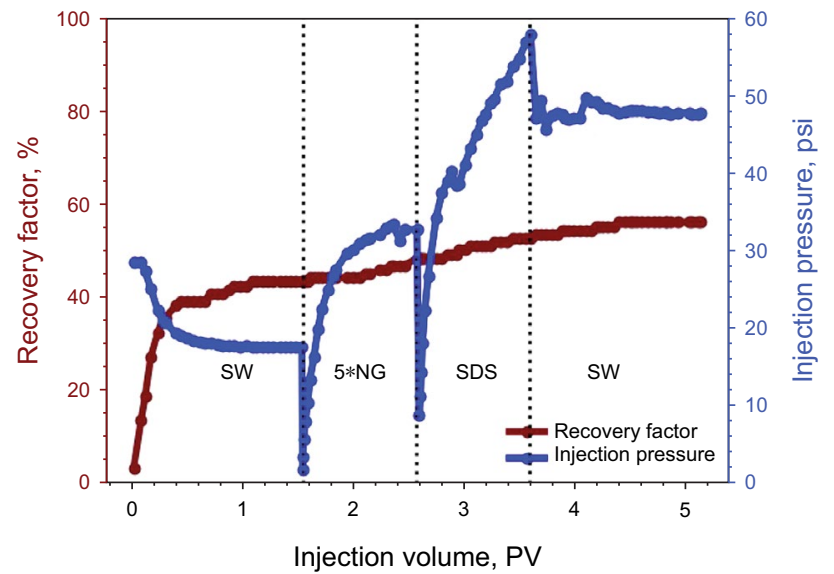

Fig. 20 Oil recovery factor and injection pressure results of core A-6 using sequential injections of $0.5 \mathrm{wt} \%$ nanogel and $0.1 \mathrm{wt} \%$ SDS followed by seawater flooding

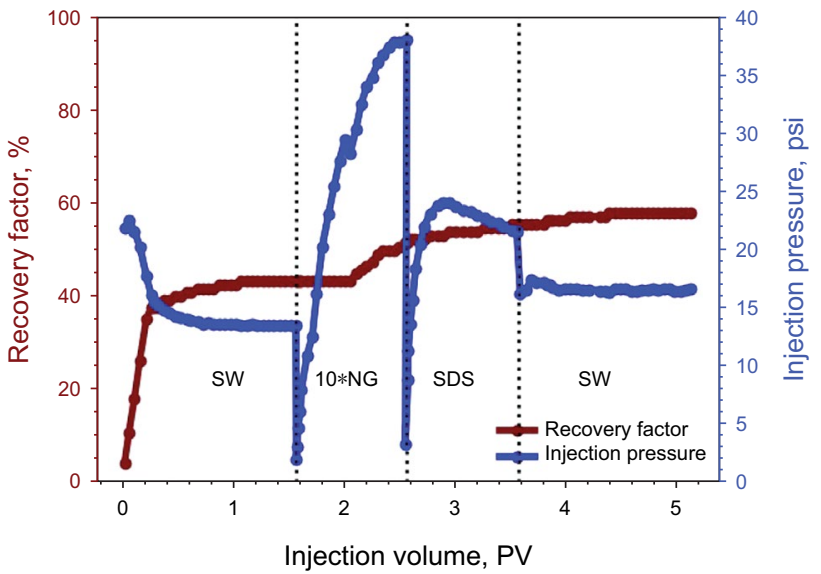

Fig. 21 Oil recovery factor and injection pressure results of core A-7 using sequential injections of $1.0 \mathrm{wt} \%$ nanogel and $0.1 \mathrm{wt} \%$ SDS followed by seawater flooding

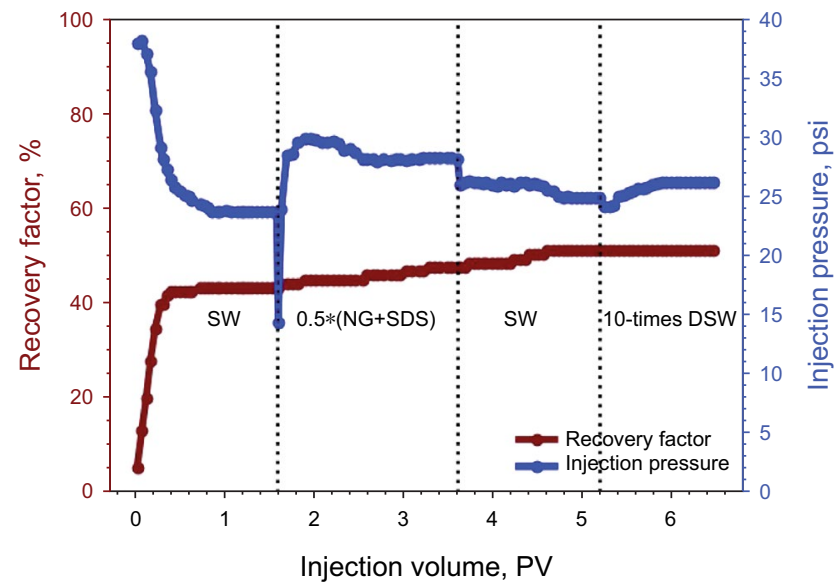

Fig. 22 Oil recovery factor and injection pressure results of core A-8 using one-slug injections of nanogel and SDS (0.05 wt\% each) followed by seawater flooding

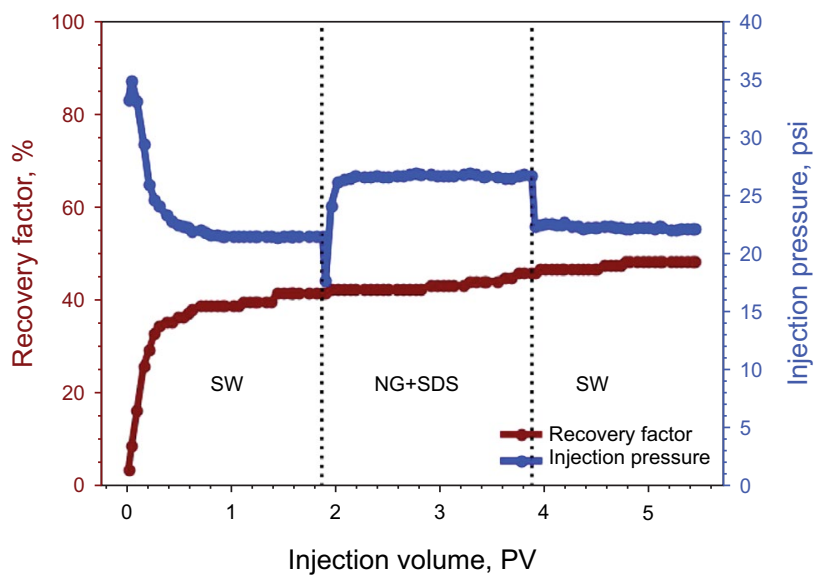

Fig. 23 Oil recovery factor and injection pressure results of core A-9 using one-slug injections of nanogel and SDS (0.1 wt\% each) followed by seawater flooding 


\section{References}

Al-Anazi HA, Sharma MM, et al. Use of a ph sensitive polymer for conformance control. In: International Symposium and Exhibition on Formation Damage Control. Society of Petroleum Engineers, 2002. https://doi.org/10.2118/73782-MS.

Almahfood M, Bai B. The synergistic effects of nanoparticle-surfactant nanofluids in eor applications. J Pet Sci Eng. 2018;171:19610. https://doi.org/10.1016/j.petrol.2018.07.030.

Almahfood MM, Bai B, et al. Potential oil recovery enhancement by a polymeric nanogel combined with surfactant for sandstone reservoirs. In: Offshore Technology Conference. Offshore Technology Conference, 2020. https://doi.org/10.4043/30601-MS.

Almohsin AM, Bai B, Imqam AH, Wei M, Kang W, Delshad M, et al. Transport of nanogel through porous media and its resistance to water flow. In: SPE Improved Oil Recovery Symposium. Society of Petroleum Engineers, 2014. https://doi.org/10.2118/16907 8-MS.

Bai B, Liu Y, Coste J-P, Li L, et al. Preformed particle gel for conformance control: transport mechanism through porous media. SPE Reserv Eval Eng. 2007;10(02):176-84. https://doi. org/10.2118/89468-PA.

Bai B, Wei M, Liu Y, et al. Field and lab experience with a successful preformed particle gel conformance control technology. In: SPE Production and Operations Symposium: Society of Petroleum Engineers; 2013. https://doi.org/10.2118/164511-MS.

Binks BP, Clint JH, Dyab AKF, Fletcher PDI, Kirkland M, Whitby CP. Ellipsometric study of monodisperse silica particles at an oil- water interface. Langmuir. 2003;19(21):8888-93. https://doi. org/10.1021/la035058g.

Binks BP, Desforges A, Duff DG. Synergistic stabilization of emulsions by a mixture of surface-active nanoparticles and surfactant. Langmuir. 2007;23(3):1098-106. https://doi.org/10.1021/la062510y.

Dong Z, Lin M, Wang H, Li M. Influence of surfactants used in surfactant-polymer flooding on the stability of Gudong crude oil emulsion. Pet Sci. 2010;7(2):263-7. https://doi.org/10.1007/s1218 2-010-0031-y.

Eskandar NG, Simovic S, Prestidge CA. Interactions of hydrophilic silica nanoparticles and classical surfactants at non-polar oil-water interface. J Colloid Interface Sci. 2011;358(1):217-25. https://doi. org/10.1016/j.jcis.2011.02.056.

Frampton H, Morgan JC, Cheung SK, Munson L, Chang KT, Williams D, et al. Development of a novel waterflood conformance control system. In: SPE/DOE Symposium on Improved Oil Recovery. Society of Petroleum Engineers, 2004. https://doi. org/10.2118/89391-MS

Geng J, Han P, Bai B, et al. Experimental study on charged nanogels for interfacial tension reduction and emulsion stabilization at various salinities and oil types. In: SPE Asia Pacific Oil and Gas Conference and Exhibition. Society of Petroleum Engineers, 2018a. https ://doi.org/10.2118/192118-MS

Geng J, Jingyang P, Wang L, Bai B. Surface charge effect of nanogel on emulsification of oil in water for fossil energy recovery. Fuel. 2018b;223:140-8. https://doi.org/10.1016/j.fuel.2018.03.046.

Green DW, Willhite GP, et al. Enhanced oil recovery, volume 6. Henry L. Doherty Memorial Fund of AIME, Society of Petroleum Engineers, 1998.

Gupta P, Elkins C, Long TE, Wilkes GL. Electrospinning of linear homopolymers of poly (methyl methacrylate): exploring relationships between fiber formation, viscosity, molecular weight and concentration in a good solvent. Polymer. 2005;46(13):4799810. https://doi.org/10.1016/j.polymer.2005.04.021.

Hendraningrat L, Li S, Torsæter O. A coreflood investigation of nanofluid enhanced oil recovery. J Pet Sci Eng. 2013;111:128-38. https ://doi.org/10.1016/j.petrol.2013.07.003.

Jiang L, Li S, Wenyang Yu, Wang J, Sun Q, Li Z. Interfacial study on the interaction between hydrophobic nanoparticles and ionic surfactants. Colloids Surf A. 2016;488:20-7. https://doi. org/10.1016/j.colsurfa.2015.10.007.

Johannessen AM, Spildo K. Enhanced oil recovery (EOR) by combining surfactant with low salinity injection. Energy Fuels. 2013;27(10):5738-49. https://doi.org/10.1021/ef400596b.

Johnson CA, Lenhoff AM. Adsorption of charged latex particles on mica studied by atomic force microscopy. J Colloid Interface Sci. 1996;179(2):587-99. https://doi.org/10.1006/jcis.1996.0253.

Lenchenkov NS, Slob M, van Dalen E, Glasbergen G, van Kruijsdijk $\mathrm{C}$, et al. Oil recovery from outcrop cores with polymeric nanospheres. In: SPE Improved Oil Recovery Conference. Society of Petroleum Engineers, 2016. https://doi.org/10.2118/179641-MS.

Moraes RR, Garcia JW, Barros MD, Lewis SH, Pfeifer CS, Liu JC, et al. Control of polymerization shrinkage and stress in nanogel-modified monomer and composite materials. Dent Mater. 2011;27(6):509-19. https://doi.org/10.1016/j.dental.2011.01.006.

Nasralla RA, Bataweel MA, Nasr-El-Din HA, et al. Investigation of wettability alteration and oil-recovery improvement by low-salinity water in sandstone rock. J Can Pet Technol. 2013;52(02):14454. https://doi.org/10.2118/146322-PA.

Pal S, Mushtaq M, Banat F, Al Sumaiti AM. Review of surfactantassisted chemical enhanced oil recovery for carbonate reservoirs: challenges and future perspectives. Pet Sci. 2018;15(1):77102. https://doi.org/10.1007/s12182-017-0198-6.

Qiu F, et al. The potential applications in heavy oil eor with the nanoparticle and surfactant stabilized solvent-based emulsion. In: Canadian unconventional resources and international petroleum conference: Society of Petroleum Engineers; 2010. https://doi. org/10.2118/134613-MS.

Rousseau D, Chauveteau G, Renard M, Tabary R, Zaitoun A, Mallo P, et al. Rheology and transport in porous media of new water shutoff/conformance control microgels. In: SPE international symposium on oilfield chemistry: Society of Petroleum Engineers; 2005. https://doi.org/10.2118/93254-MS.

Suleimanov BA, Veliyev EF. Novel polymeric nanogel as diversion agent for enhanced oil recovery. Pet Sci Technol. 2017;35(4):319_ 26. https://doi.org/10.1080/10916466.2016.1258417.

Sun X, Zhang Y, Chen G, Gai Z. Application of nanoparticles in enhanced oil recovery: a critical review of recent progress. Energies. 2017;10(3):345. https://doi.org/10.3390/en10030345.

Thomas S. Enhanced oil recovery-an overview. Oil Gas Sci Technolo-Revue de l'IFP. 2008;63(1):9-19. https://doi.org/10.2516/ ogst:2007060.

Wagner NJ, Brady JF. Shear thickening in colloidal dispersions. Phys Today. 2009;62(10):27-32. https://doi.org/10.1063/1.3248476.

$\mathrm{Xu} \mathrm{Z,} \mathrm{Li} \mathrm{S,} \mathrm{Li} \mathrm{B,} \mathrm{et} \mathrm{al.} \mathrm{A} \mathrm{review} \mathrm{of} \mathrm{development} \mathrm{methods} \mathrm{and} \mathrm{EOR}$ technologies for carbonate reservoirs. Pet. Sci. 2020;17(5):990_ 1013. https://doi.org/10.1007/s12182-020-00467-5.

Yousef AA, Al-Saleh SH, Al-Kaabi A, Al-Jawfi MS, et al. Laboratory investigation of the impact of injection-water salinity and ionic content on oil recovery from carbonate reservoirs. SPE Reserv Eval Eng. 2011;14(05):578-93. https://doi.org/10.2118/13763 4-PA. 